\title{
Causality change between Korea and other major equity markets
}

\author{
Tae Yeon Kwon ${ }^{1, a}$ \\ ${ }^{a}$ Department of International Finance, Hankuk University of Foreign Studies, Korea
}

\begin{abstract}
The world financial markets are inter-linked in ways that varies according to market and time. We examine the causality of change focusing on the Korean market as related to the U.S. (S\&P 500), Japan (Nikkei 225), Hong-Kong (HSI), and European (DAX) markets. In order to capture time-varying causality running from and to the Korea stock market, we apply the Granger causality test under a VAR model with a wild bootstrap rollingwindow approach. We also propose a new concept of a significant causality ratio to measure the intensity of the Granger causality in each time unit. There are many asymmetric strengths in mutual Granger causal relationships. Moreover, there are cases with significant Granger causal relations only in one direction. The period with the most severe Granger causality both running from and to the KOSPI market is the GFC. The market that formed the two-way Granger causal relationship with the KOSPI market for the longest period is the S\&P 500. The HSI and DAX markets have the strongest two-way Granger causal relationship with the KOSPI shortly after 2000, and the Nikkei market had the strongest two-way Granger causal relationship with the KOSPI market before the Asian financial crisis.
\end{abstract}

Keywords: Granger causality test, wild bootstrap, rolling-window, significant causality ratio, KOSPI

\section{Introduction}

Inter-dependence among world financial markets has grown due to advances in technology and there have been several studies about the comovement and causal relationship between stock markets (Baek and Oh, 2016; Kim and Bang, 2014; Rapach et al., 2013; Ajmi et al., 2014; Masih and Masih, 1999; Smith et al., 1993). However, it is difficult to assume that the relationship between different stock markets is constant over time. For example, the inter-linkage among world financial markets were augmented during the Global Financial Crisis (GFC). Throughout history, a world-wide stock market crash has magnified the inter-dependence of financial markets.

In this respect, several studies have discussed the time-varying aspect of the inter-linkage of financial markets. Engle (2002) and Kim (2014) adopted a method to model dynamic conditional correlation based on GARCH-typed model. Masih and Masih (2001) examined short- and long-run (cointegration) relations among international stock markets. Masih and Masih (1999) adopt a generalized variance decomposition analysis to reflect the dynamic property of causal relationships among world financial markets. Eun and Shim (1989) show the stock market co-movement by estimating a nine-market (Australia, Canada, France, Germany, Hong Kong, Japan, Switzerland, United Kingdom, and U.S.) vector autoregression (VAR) system. Smith et al. (1993) showed the brief periods

\footnotetext{
${ }^{1}$ Department of International Finance, Hankuk University of Foreign Studies, 81 Oedae-ro, Mohyeon-eup, Cheoin-gu, Yongin-si, Gyeonggi-do 17035, Korea. Email: tykwon@hufs.ac.kr

Published 31 July 2018 / journal homepage: http://csam.or.kr (c) 2018 The Korean Statistical Society, and Korean International Statistical Society. All rights reserved.
} 
of causality running from and to U.S. by conducting Granger causality tests for a series of rollingwindow subsamples.

In this paper, we discuss the inter-linkage between Korea and other major equity markets. There are several studies about the inter-linkages of stock markets related to Korean market. Kim and Rogers (1995) examined the relationship between the stock markets in Korea (KOSPI), Japan (Nikkei 225), and the United States (S\&P 500) between 1985 and 1992 based on GARCH model. They showed that the volatility spillovers from the Japan and U.S. markets in the determination of KOSPI returns and volatilities. Kim (2005) found the significant dynamic information spillover effects from the U.S. in all the Asia-Pacific markets, but Japanese information flows were relatively weak. Yang and Lim (2004) discussed the issue of stock market contagion among Korea, Hong Kong, Indonesia, Malaysia, Thailand, Philippines, Singapore, Taiwan, and Japan from 1990 to 2000. Based on Granger causality test under the VAR model during this period, Korean stock market are inter-linked with Hong Kong and Japan before and after the 1997 Asian financial crisis.

In this paper, we examine the change of Granger causality running from and to the Korea equity market. We conduct a time varying Granger causality test between the Korea Composite Stock Price Index (KOSPI) and other major equity markets from January 1981 to November 2017 (monthly data). We test pairwise causality between the KOSPI and the S\&P 500, Nikkei 225, Hong Kong Hang Seng Index (HSI), and Deutscher Aktienindex (DAX).

In addition to being a causality study that focuses on the Korean market, this paper also has the following contributions in terms of data analysis methods. In recent studies of causality between stock markets, the wild bootstrap method (Hafner and Herwartz, 2009) has been applied for a robust $p$-value calculation under conditional heteroskedasticity and Stambaugh (1999)'s bias (Rapach et al., 2013; Ajmi et al., 2014).

We apply the wild bootstrap method combined with a rolling window approach. In this paper, we use the wild bootstrap Granger causality test under the VAR model for a series of rolling window sub-sample. We consider different rolling window sizes from 3 to 15 years. We compare the results according to a range of rolling window sizes as well as determine the most appropriate rolling window size based on prediction error. The wild bootstrap method can also solve the problem due to small sample size as discussed in Mantalos (2000).

For efficient summary of time varying causality, we propose a new concept called the significant causality ratio (SCR). Previous studies of causality changes based on the rolling window method (Nyakabawo et al., 2015; Smith et al., 1993) have summarized the significance in each time window as if it were the result of only the beginning, middle, or end point in the time window. It is hard to compare the results with different rolling window sizes. Moreover, it is impossible to detect the effect of a certain month in significant causality. However, the SCR proposed in this paper can be interpreted as an indicator to measure how strong the Granger causal relationship is between two stock markets in a certain month.

Empirical results show that the direction of Granger causality between two stock markets is not always symmetric and the significant (or insignificant) causality during the entire time period (January 1981 to November 2017) does not lead to significant (or insignificant) short term causality. The period of highest mutual causality is the GFC period. The market that formed the two-way Granger causal relationship with the KOSPI market for the longest period is the U.S. S\&P 500. Some periods with only one way Granger causality with the KOSPI market appeared in the Japan, Europe, and Hong Kong markets. In the 2000s, the two-way Granger causal relationship with the KOSPI increased in the Hong Kong (HSI) and Europe (DAX) markets. However, the Japan (Nikkei 225) market had the strongest two-way Granger causal relationship with the KOSPI market before the Asian financial 
crisis.

This paper consists of five chapters. In Section 2, we describe the data and the methods for Granger causality test with wild bootstrap method. Section 3 discuss the rolling window method and window size selection procedure and results. Section 4 introduces the SCR and shows the results fitted based on the Granger causality test with wild bootstrap and rolling window approaches and summarized based on SCR. Some concluding remarks are included in Section 5.

\section{The basic statistical methods and data}

The statistical methods adopted to analyze the data in this paper can be summarized by: (1) the Granger causality test under VAR model, (2) the wild bootstrap method for the robust $p$-value calculation, and (3) the rolling-window approach in order to capture time varying causality. We first address (1) and (2) in this section and (3) will be discussed in more detail in Section 3.

\subsection{The Granger causality test with the wild bootstrap}

This paper analyzes the Granger causality (Granger, 1969) between two different countries' stock markets under the VAR model. McMillin (1988) and Masih and Masih (1999) point out that VAR models are useful in testing Granger causality. Consider the following bivariate $\operatorname{VAR}(p)$ model for two processes $x_{t}$ and $y_{t}$.

$$
\left[\begin{array}{l}
y_{t} \\
x_{t}
\end{array}\right]=\left[\begin{array}{l}
\phi_{10} \\
\phi_{20}
\end{array}\right]+\left[\begin{array}{ll}
\phi_{11,1} & \phi_{12,1} \\
\phi_{21,1} & \phi_{22,1}
\end{array}\right]\left[\begin{array}{l}
y_{t-1} \\
x_{t-1}
\end{array}\right]+\cdots+\left[\begin{array}{ll}
\phi_{11, p} & \phi_{12, p} \\
\phi_{21, p} & \phi_{22, p}
\end{array}\right]\left[\begin{array}{l}
y_{t-p} \\
x_{t-p}
\end{array}\right]+\left[\begin{array}{l}
\epsilon_{1 t} \\
\epsilon_{2 t}
\end{array}\right] .
$$

The null hypothesis that the $x_{t}$ not being the Granger-cause for $y_{t}$ is defined as $\phi_{12, k}=0$ for all $k=1, \ldots, p$ in equation (2.1). The alternative is $\exists \phi_{12, k} \neq 0$ for $k=1, \ldots, p$ in equation (2.1). The test statistics for the $F$-type Granger causality test are distributed as $F(p, 2 T-n)$, where $T$ is time duration and $n$ is the total number of parameters in equation (2.1).

We adopt a wild bootstrap method to calculate the $p$-value of the Granger causality test. Mantalos (2000) shows that the $p$-value of the Granger causality test performs poorly in small and medium sized samples. However, they showed that the residual based bootstrap test possesses the best power and size properties regardless of cointegration properties. We use the wild bootstrap method (one of the residual based bootstrap methods) to endure the small sample size in order to adopt the rolling window approach (discussed in Section 3). Hafner and Herwartz (2009) introduce a wild bootstrap method to test parameter restriction in VAR models that include a Granger causality test in the VAR model. They show that the wild bootstrap method is robust under the conditional heteroscedasticity of an unknown form.

The wild bootstrap method proceeds as follows.

Step 1. Estimate $\operatorname{VAR}(\mathrm{p})$ and residual $\hat{\epsilon}_{t}=\left(\hat{\epsilon}_{1 t}, \hat{\epsilon}_{2 t}\right)$ in equation (2.1) with and without restriction $\left(H_{0}\right)$.

Step 2. Calculate the test-statistics $F$ for the Granger causality test.

Step 3. Generate bootstrap sample of $\epsilon_{t}^{*}=\hat{\epsilon}_{t} \eta_{t}$, where $\eta_{t}$ is sampled independently from identical distribution with mean 0 and variance 1 .

Step 4. Estimate equation (2.1) with each artificial series and compute the test-statistics $F^{*}$. 
Step 5. Repeat the bootstrap iteration $M$ for Steps 3 and 4 . We then can obtain $M$ test-statistics $F_{1}^{*}, \ldots, F_{M}^{*}$.

Step 6. Compute the bootstrap $p$-values $P_{\text {boots }}$ as follows

$$
P_{\text {boots }}=\frac{1}{M} \sum_{m=1}^{M} I\left(F_{m}^{*}>F\right),
$$

where $I(\cdot)$ is an indicator function, such that $I(\cdot)$ is 1 if $(\cdot)$ is true and 0 otherwise.

Detailed discussion on the residual based bootstrap method and the wild bootstrap methods are in Rapach et al., (2013), Ajmi et al., (2014), and Nyakabawo et al. (2015).

\subsection{Data}

The log return of the representative stock indexes of five countries (Korea, U.S., Japan, Germany, and Hong-Kong, which are KOSPI, S\&P 500, Nikkei 225, DAX, and HSI respectively) are tested from January 1981 to November 2017. Among several major equity market indexes, we limit the indexes whose values from January 1981 to November 2017 are all available. We collect the monthly indexes from Yahoo finance and the Economic Statistics System of Bank of Korea.

We use the mean of the daily closing index for the monthly values for KOSPI, S\&P 500, and Nikkei 225, but we use the closing value of each month for DAX and HSI due to the limitations of data availability (Different from KOSPI, S\&P500, and Nikkei 225, there could be un-adjusted month end effect in DAX and HSI because we use the closing value of each month for them. However, we use the return instead of the index value itself, so we can expect that the month end effects are offset by each other.) In order to detect the Granger causality during long periods, monthly data is usually used (Rapach et al., 2013; Masih and Masih, 1999), because of the noise and holiday effect in daily data (Smith et al., 1993). We use the series of monthly $\log$ return $r_{t}$ which is given below.

$$
r_{t}=\ln \left(p_{t}\right)-\ln \left(p_{t-1}\right)
$$

where $p_{t}$ is the price of stock.

Figure 1 shows the time plot of major markets' log return with the log return of the KOSPI market marked with a red dotted line in each plot. Table 1 shows the summary of log return series of KOSPI, S\&P 500, Nikkei 225, DAX, and HSI. We test for the existence of unit root in return series based on an augmented Dickey-Fuller test (ADF) and a Phillips-Perron (PP) test. The results indicate that the null hypothesis of the unit root is rejected at the level of a $1 \%$ significant level for all series. The stationary condition of $\log$ return series $r_{t}$ is satisfied for further statistical analysis.

\subsection{Results of full sample Granger causality}

We test the null hypothesis that the log return of KOSPI does not Granger cause the log return of S\&P 500, Nikkei DAX, or HSI (and vice versa) based on the entire sample period of 1981 and 2017. We set a bivariate $\operatorname{VAR}(p)$ model for all possible pairs between KOSPI and other indexes (S\&P 500, Nikkei 225, DAX, and HSI). The order of the VAR model is selected based on Akaike information criterion (AIC). The bootstrap sample is taken with 1,000 repetitions.

Table 2 shows $F$-test statistics and corresponding $p$-values with 1,000 bootstrap runs. The results indicate that log returns of S\&P500, DAX, and HSI Granger cause the log return of KOSPI, while the $\log$ return of KOSPI only Granger causes the log return of Nikkei 225. We conclude only one-way causality running from the KOSPI to Nikkei and from S\&P 500, DAX, and HSI to KOSPI. 

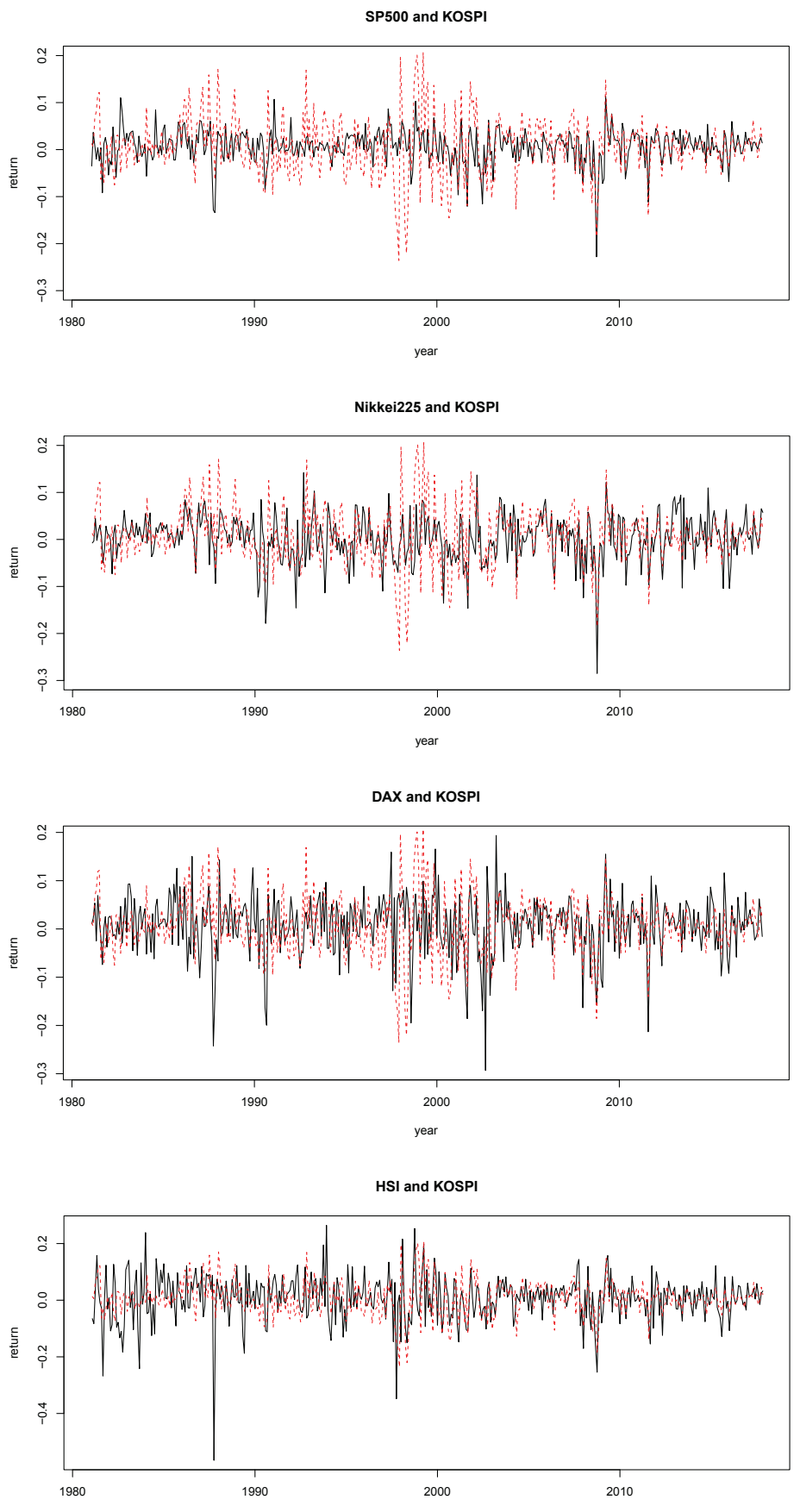

Figure 1: Time plot of the log return of S\&P500, Nikkei 225, DAX, and HSI with that of KOSPI. The red dotted line is log return of KOSPI market. The black solid line is the log return of S\&P500, Nikkei 225, DAX, or HSI, respectively. 
Table 1: Summary of log return, January 1981 to November 2017

\begin{tabular}{ccccccc}
\hline \hline & Mean & Min & Max & Std.dev & ADF-type 3 $(p$-value $)$ & PP $(p$-value $)$ \\
\hline KOSPI & 0.0072 & -0.2358 & 0.2072 & 0.0614 & $-6.3442(<0.01)$ & $-14.6811(<0.01)$ \\
SP500 & 0.0067 & -0.2281 & 0.1135 & 0.0358 & $-6.8794(<0.01)$ & $-16.0565(<0.01)$ \\
NIKKEI225 & 0.0026 & -0.2850 & 0.1422 & 0.0493 & $-6.7559(<0.01)$ & $-16.3808(<0.01)$ \\
DAX & 0.0075 & -0.2933 & 0.1937 & 0.0600 & $-7.0972(<0.01)$ & $-19.6658(<0.01)$ \\
HSI & 0.0066 & -0.5656 & 0.2645 & 0.0801 & $-7.8227(<0.01)$ & $-19.7371(<0.01)$ \\
\hline \hline
\end{tabular}

This table presents the summary statistics of data. Augmented Dickey-Fuller test statistics (ADF) and Philips-Perron test statistics (PP) are also given with the $p$-value in parenthesis.

Table 2: Full sample (January, 1981-November, 2017) Granger causality tests, where $H_{0}: x$ does not Granger cause $y$

\begin{tabular}{cccc}
\hline \hline$y$ & $x$ & $F$-statistics & $p$-value \\
\hline KOSPI & SP500 & 4.988 & 0.028 \\
SP500 & KOSPI & 0.835 & 0.471 \\
KOSPI & Nikkei & 1.791 & 0.188 \\
Nikkei & KOSPI & 3.973 & 0.005 \\
KOSPI & DAX & 7.708 & 0.012 \\
DAX & KOSPI & 0.335 & 0.756 \\
KOSPI & HSI & 22.214 & 0.001 \\
HSI & KOSPI & 5.217 & 0.057 \\
\hline \hline
\end{tabular}

\section{Selection of rolling window size}

In previous studies analyzing the Granger causality, Smith et al. (1993) used 3-years of rolling window data, Singh and Kishor (2017) used 10 years of data and Masih and Masih (1999) used 5.5 years of data.

In this paper, we analyze the time-series data with a rolling window approach in order to capture change and time-varying causality. First, the Granger causality is estimated for the first $m$ observation, where $m$ is window size. We then drop the one observation that is the oldest, while adding one new observation and reestimate the relationship.

We consider window sizes of 3 to 15 years and compare the results. A small window size leads the small subsample to reduce the heterogeneity within a subsample and improve the representativeness. However, a large standard error of parameter estimates, due to the small sample size, reduce the estimation accuracy. In addition, a large window size improves accuracy, but reduces the representativeness (Pesaran and Timmermann, 2005; Nyakabawo et al., 2015). We need to select the window size that balances this trade-off.

After estimating the $\operatorname{VAR}(p)$ model in equation (2.1), where VAR order $p$ is determined based on AIC, we calculate the mean squared prediction error (MSPE) in predicting $y_{t}$ with $x_{t}$. Table 3 shows the MSPE in predicting $y_{t+1}$ (1-month ahead log return $y$ at time $t$ ) with $x_{t}$ and $y_{t+6}$ (6-month ahead $\log$ return $y$ at time $t$ ) with $x_{t}$, respectively. All procedures for model identification and estimation are conducted independently in every different time window.

MSPE decrease (accuracy increase) as window size increases, but the degree of MSPE decline is negligible after a 5-year window size. If there is no (or negligible) difference in the prediction accuracy, choosing a window size as small as possible is good in terms of representativeness. We choose a 5-year window size for balancing the two values of representativeness and accuracy in model estimation. 
Table 3: Mean squared prediction error in predicting 1-month and 6-month ahead log return of $y$ with $x_{t}$

\begin{tabular}{|c|c|c|c|c|c|c|c|c|c|c|}
\hline \multirow{2}{*}{ Predict } & \multirow[b]{2}{*}{$y_{t}$} & \multirow[b]{2}{*}{$x_{t}$} & \multicolumn{8}{|c|}{ Window size } \\
\hline & & & 3 & 3.5 & 4 & 4.5 & 5 & 7 & 10 & 15 \\
\hline \multirow{8}{*}{ 1-month } & KOSPI & SP500 & 0.0598 & 0.0932 & 0.0156 & 0.0066 & 0.0051 & 0.0042 & 0.0044 & 0.0039 \\
\hline & SP500 & KOSPI & 0.0299 & 0.0409 & 0.0054 & 0.0019 & 0.0016 & 0.0013 & 0.0013 & 0.0014 \\
\hline & KOSPI & Nikkei & 0.0801 & 0.1024 & 0.0134 & 0.0053 & 0.0043 & 0.0038 & 0.0039 & 0.0040 \\
\hline & Nikkei & KOSPI & 0.0874 & 0.1219 & 0.0080 & 0.0038 & 0.0031 & 0.0027 & 0.0027 & 0.0026 \\
\hline & KOSPI & DAX & 0.0683 & 0.0863 & 0.0115 & 0.0070 & 0.0046 & 0.0038 & 0.0038 & 0.0039 \\
\hline & DAX & KOSPI & 0.0813 & 0.1138 & 0.0175 & 0.0085 & 0.0051 & 0.0041 & 0.0039 & 0.0041 \\
\hline & KOSPI & HS & 0.0611 & 0.0913 & 0.0121 & 0.0060 & 0.0052 & 0.0037 & 0.0037 & 0.0039 \\
\hline & HS & KOSPI & 0.1116 & 0.1497 & 0.0195 & 0.0088 & 0.0079 & 0.0058 & 0.0055 & 0.0051 \\
\hline \multirow{8}{*}{ 6-month } & KOSPI & SP500 & 243 & 35 & 0.0231 & 0.0065 & 0.0047 & 0.0045 & 0.0044 & 0.0044 \\
\hline & SP500 & KOSPI & 102 & 18 & 0.0090 & 0.0021 & 0.0016 & 0.0013 & 0.0013 & 0.0014 \\
\hline & KOSPI & Nikkei & 11,087 & 4,075 & 0.0133 & 0.0053 & 0.0045 & 0.0041 & 0.0042 & 0.0045 \\
\hline & Nikkei & KOSPI & 11,110 & 2,573 & 0.0084 & 0.0037 & 0.0031 & 0.0028 & 0.0028 & 0.0027 \\
\hline & KOSPI & DAX & 417 & 1,163 & 0.0190 & 0.0079 & 0.0051 & 0.0042 & 0.0042 & 0.0044 \\
\hline & DAX & KOSPI & 95 & 550 & 0.0190 & 0.0082 & 0.0049 & 0.0040 & 0.0037 & 0.0041 \\
\hline & KOSPI & $\mathrm{HS}$ & 1.13 & 429,574 & 0.0135 & 0.0066 & 0.0053 & 0.0042 & 0.0042 & 0.0045 \\
\hline & HS & KOSPI & 9.25 & 3,975 & 0.0181 & 0.0089 & 0.0075 & 0.0054 & 0.0053 & 0.0051 \\
\hline
\end{tabular}

\section{The significant causality ratio}

Several structural changes in financial markets between 1981 and 2017 do not allow the long-term causal relationship in Table 2 to remain a short-term causal relationship. The rolling-window Granger causality test is motivated by this perspective. In this paper, we propose a new descriptive statistics called SCR to summarize the intensity of Granger causality in each time point and describe the time varying causality effectively.

\subsection{Meaning of SCR and its calculation}

Previous studies of causality changes based on the rolling window method (Nyakabawo et al., 2015; Smith et al., 1993) summarize the $p$-value or significance of the Granger causality in each time window. They denote the results as if they were at the beginning, middle, or end of each time window. Therefore, it is hard to compare the results with different rolling window sizes. Furthermore, it is impossible to examine the intensity of causality of a certain month.

We propose a new concept named SCR for each month, which is the percentage of time windows with significant Granger causality among all time windows that include the month and defined as:

$$
\mathrm{SCR}_{t}=\frac{\mathrm{STW}_{t}}{\mathrm{TTW}_{t}}
$$

where $\mathrm{STW}_{t}$ is the number of time windows with a significant Granger causality time that include $t^{\text {th }}$ month and with $\mathrm{TTW}_{t}$ as the total number of time windows including $t^{\text {th }}$ month. We set 0.05 as significance level and $p$-value is calculated with 1,000 times bootstrap runs.

$\mathrm{SCR}_{t}$ is the ratio of time intervals with significant causality among all possible datasets that can be made with equal length sequences that contain a particular time $t$. In this paper, $\mathrm{SCR}_{t}$ is proposed as the descriptive statistics that make the comparison of the intensity of causality possible. If the $\mathrm{SCR}_{t}$ is close to 1 , it can be interpreted that the intensity of causality at time $t$ is high because it shows a significant causality regardless of the composition of the sequence containing $t$. Conversely, $\mathrm{SCR}_{t}$ near zero implies that the intensity of causality is weak because the significance strongly depends on how the sequence containing $t$ is constructed. 
Table 4: Summary of SCR with different $\phi_{12,1}$ in 1,000 simulated bivariate VAR(1) paths

\begin{tabular}{cccccc}
\hline \hline$\phi_{12,1}$ & 0 & 0.1 & 0.2 & 0.3 & 0.5 \\
\hline Mean (and s.d.) of bootstrap $p$-value & 0.509 & 0.330 & 0.101 & 0.018 & 0.000 \\
for Granger causality & $(0.286)$ & $(0.295)$ & $(0.175)$ & $(0.051)$ & $(0.001)$ \\
\hline Mean of SCR & 0.051 & 0.123 & 0.328 & 0.613 & 0.944 \\
S.d. of SCR & 0.121 & 0.206 & 0.332 & 0.341 & 0.145 \\
5\% quantile of SCR & 0.000 & 0.000 & 0.000 & 0.000 & 0.617 \\
95\% quantile of SCR & 0.333 & 0.600 & 0.967 & 1.000 & 1.000 \\
\hline \hline
\end{tabular}

SCR = significant causality ratio.

In order to show that how $\mathrm{SCR}_{t}$ reflect the intensity of causality, we perform simple simulation study. We simulate bivariate $\operatorname{VAR}(1)$ paths during 120 months as given in equation (4.2) with $\phi_{12,1}=$ $0,0.1,0.2,0.3$, and 0.5 , respectively. We simulate 1,000 hypothetical datasets for each $\phi_{12,1}$ value and $\epsilon_{1 t}$ and $\epsilon_{2 t}$ are simulated from independent $\operatorname{Normal}(0,1)$ distribution.

$$
\left[\begin{array}{l}
y_{t} \\
x_{t}
\end{array}\right]=\left[\begin{array}{cc}
0.3 & \phi_{12,1} \\
0 & 0.3
\end{array}\right]\left[\begin{array}{l}
y_{t-1} \\
x_{t-1}
\end{array}\right]+\left[\begin{array}{l}
\epsilon_{1 t} \\
\epsilon_{2 t}
\end{array}\right] .
$$

SCR at $60^{\text {th }}$ month with 60 months (5 years) time window is then calculated for each simulated dataset and the results are summarized in Table 4 . We can find that as the degree of influence (significance) of $x_{t-1}$ on $y_{t}$ increases, the SCR value increases.

SCR makes it possible to compare the intensity of causality in each month regardless of window size since the SCR is designed to be calculated at all time points. In addition, SCR can be extended to other types of time-series models that determine significance based on $p$-value.

\subsection{Empirical results}

Figure 2 shows the $\mathrm{SCR}_{t}$ from December, 1985 to November, 2012, which test the Granger causality from the S\&P 500, Nikkei, DAX, and HS to KOSPI. Figure 3 shows the $\mathrm{SCR}_{t}$ from December, 1985 to November, 2012, which test Granger causality from KOSPI to S\&P 500, Nikkei, DAX, and HSI. We use all data from January 1981 to November 2017 in the analysis; however, the results before December 1985 and after November 2012 are not summarized in these figures because the total number of time windows containing them (which is the denominator of SCR) is small.

Figure 2 and Figure 3 present the results with a 5-year rolling window; in addition, Figure 4 and Figure 5 show the results based on a 7-year rolling window size and give similar conclusions about the direction and significance of the Granger causality between different stock markets.

During the GFC (which is around 2008) we can find the most severe Granger causality running from and to the KOSPI market. There is a slight difference in the intensities measured with SCR, but the Granger causal relationship between the U.S. S\&P 500 market and Korea's KOSPI market is generally two-way. However, there are more asymmetric cases than symmetric cases in Granger causality measured with SCR between Nikkei, DAX, HSI, and KOSPI.

At the end of 1995, the degree of Granger causality from the Nikkei market to the KOSPI market was 0.12 , while the Granger causality in the opposite direction was 0.6. In particular, the asymmetry of the Granger causal relationship with the KOSPI market is severe in the DAX and HSI markets in 1998 during the Asian financial crisis. From 1998 to mid-2006, the strength of Granger causality running from DAX and HSI to KOSPI continued to increase, reaching a SCR of 0.8 , but in the opposite direction, the degree remained below 0.1 .

It is the U.S. market (S\&P 500) that brought the longest period of mutual causality with the Korean market (KOSPI). In terms of SCR, Hong Kong has the greatest impact on Korea and is also 

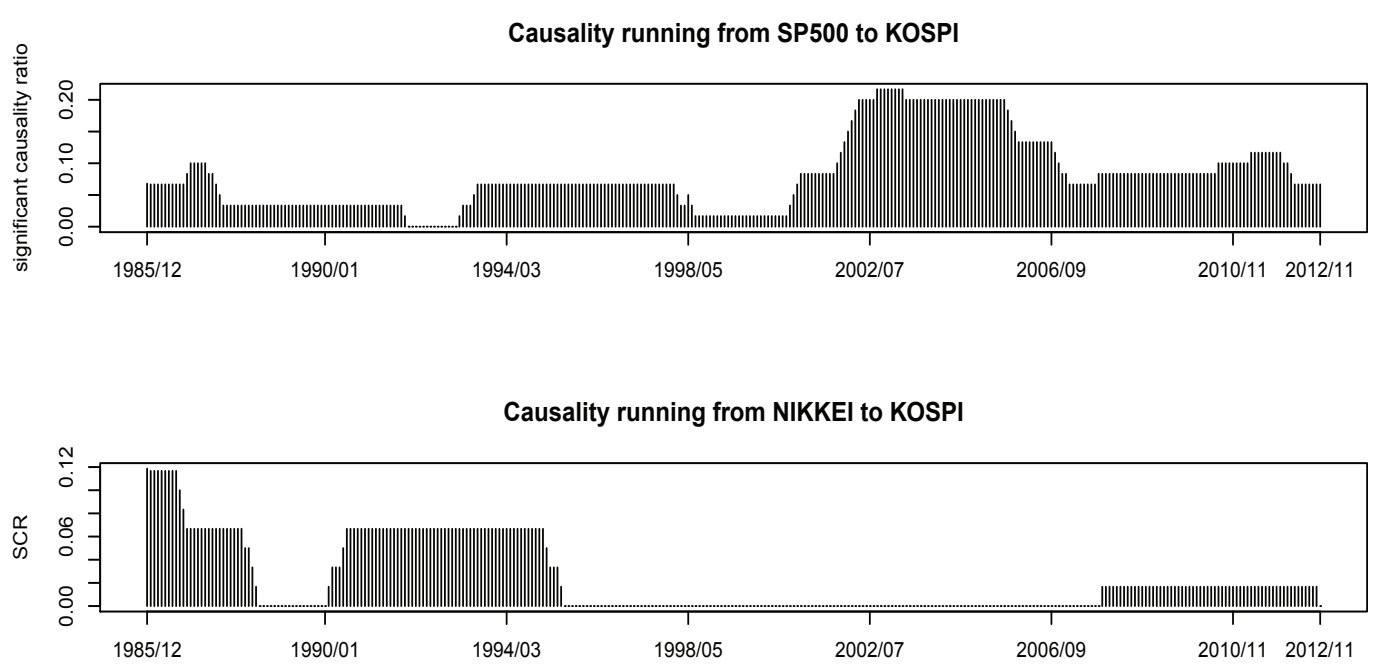

Causality running from DAX to KOSPI

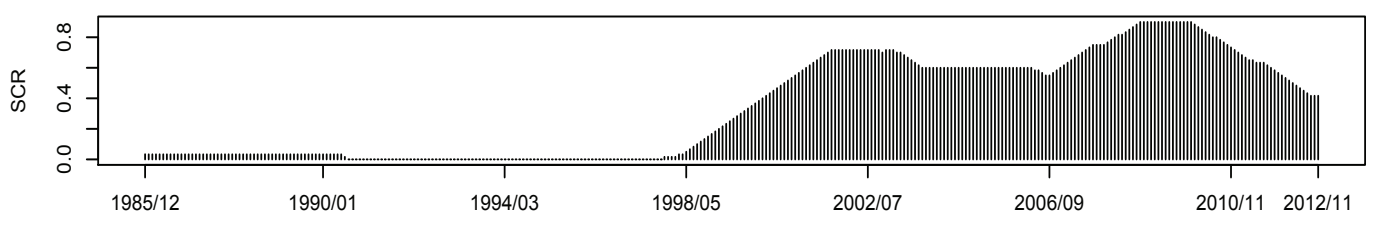

Causality running from HS to KOSPI

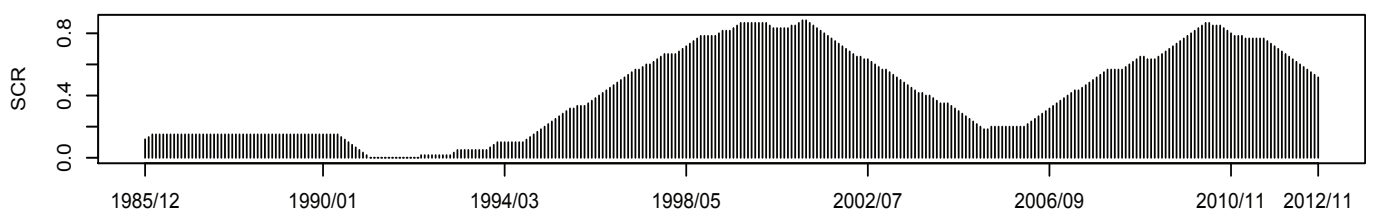

Figure 2: Causality running to KOSPI with a 5-year rolling window size. These graphs show the significant causality ratio that run from major stock markets to the KOSPI in each month.

the country most affected. Japan has the shortest period of significant Granger causality running to Korea, among the four countries, but Japan was the most influential country to Korea before mid-1990 when the Asian financial crisis occurred. However, the degree of Japan's Granger cause to KOSPI has since dropped sharply and Europe and Hong Kong have continued to increase the degree of their Granger cause to KOSPI. In the 2000s, Granger causality which is running from KOSPI to JAPAN also dropped sharply. However, Granger causality running from KOSPI to Hong Kong and Europe sored.

The short-term results are quite different from the long-term results (Table 2). We find that the 


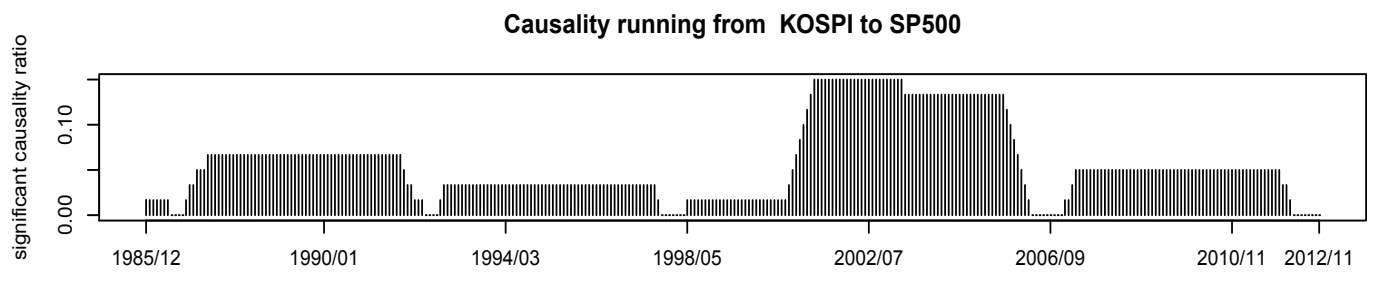

Causality running from KOSPI to NIKKEI

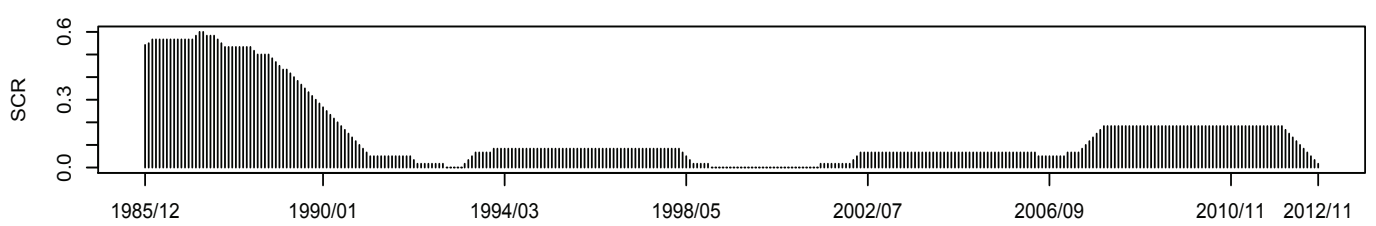

Causality running from KOSPI to DAX

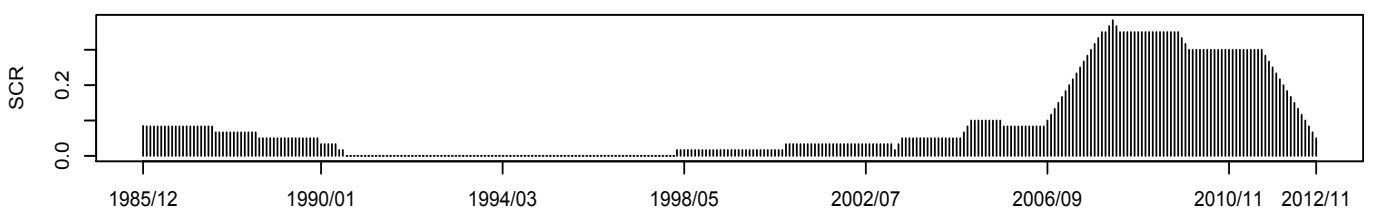

Causality running from KOSPI to HS

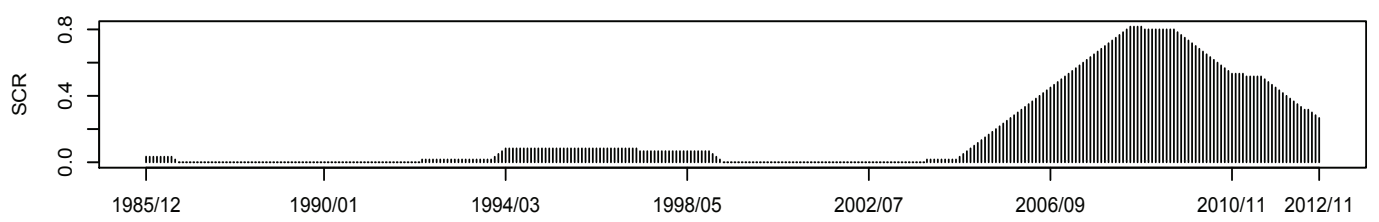

Figure 3: Causality running from KOSPI with a 5-year rolling window size. These graphs show the significant causality ratio that run from the KOSPI to stock markets in each month.

inter-linkage between stock markets is not a problem that can be explained with only one $p$-value. The intensity of Granger causality fluctuates differently in different markets. The degree to which shocks affect causality varies from market to market.

\section{Conclusion}

In this paper, we examine changes in the Granger causality between the KOSPI market and other major stock market returns from 1981 to 2017 using a bootstrap rolling window method. We have determined appropriate window size balancing accuracy in model estimation and model representa- 


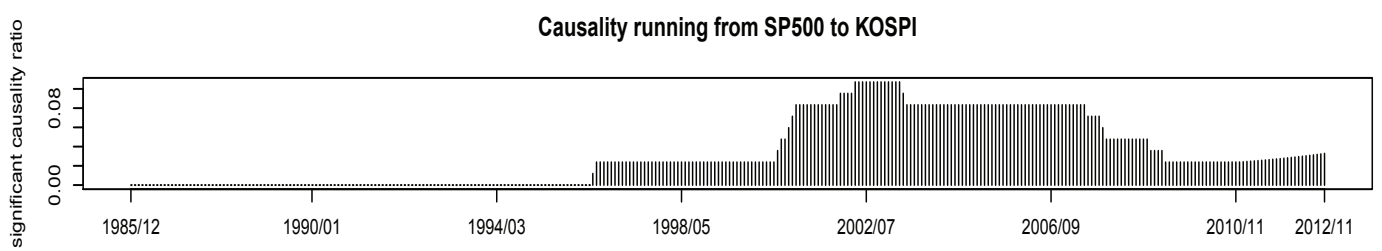

Causality running from NIKKEI to KOSPI

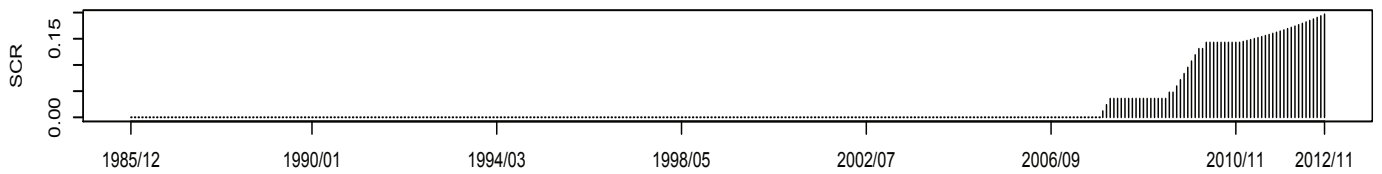

Causality running from DAX to KOSPI

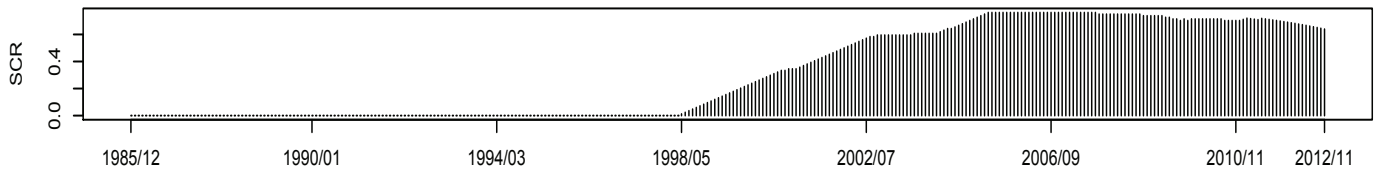

Causality running from HS to KOSPI

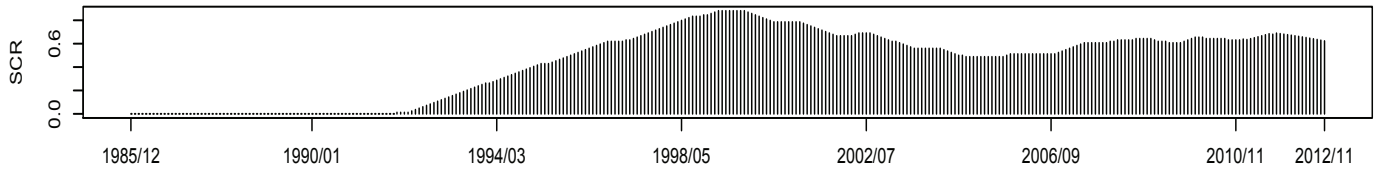

Figure 4: Causality running to KOSPI with a 7-year rolling window size. The graphs show the significant causality ratio that run from major stock markets to the KOSPI in each month.

tiveness. As a measure for intensity of Granger causality, we propose the SCR which is the percentage of time windows with significant Granger causality among all time windows included in a month. The SCR makes it possible to compare the intensity of causality in each month regardless of window size.

With the new methods presented above, we examined the Granger causality running from and to the KOSPI market by country and by period. The short-term results are quite different from longterm results. During the GFC, we can find the most severe Granger causality running from and to the KOSPI market. The market that formed the two-way Granger causal relationship with the KOSPI market for the longest period is the U.S. S\&P 500 market. Several periods with asymmetric or even one-way Granger causality with the KOSPI market appeared in the Japan, Europe, and Hong Kong markets. In the 2000s, the two-way Granger causal relationship increased in the HSI and DAX; however, the Nikkei market had the strongest two-way Granger causal relationship with the KOSPI market before the Asian financial crisis. 


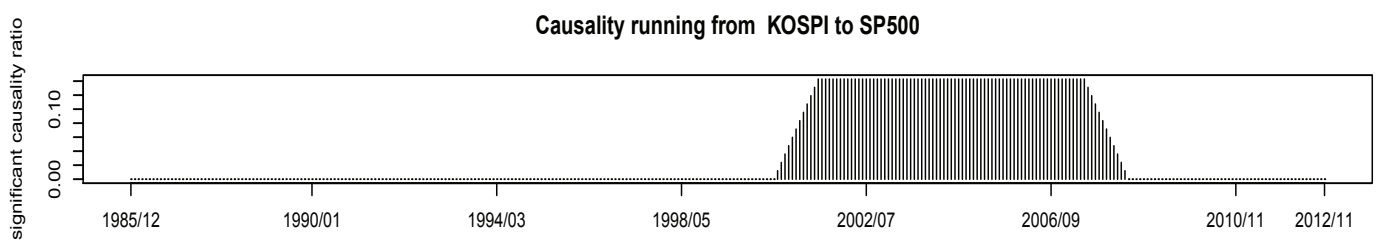

Causality running from KOSPI to NIKKEI

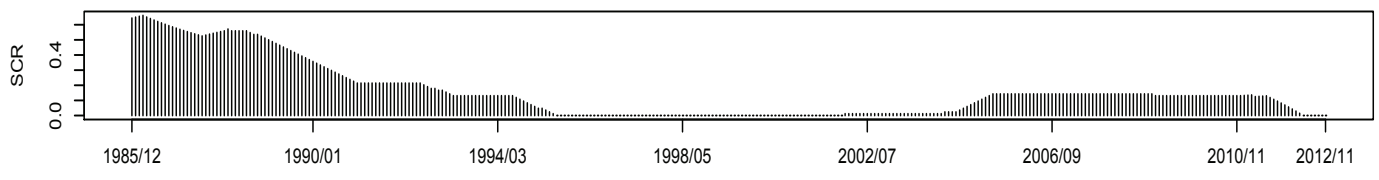

Causality running from KOSPI to DAX

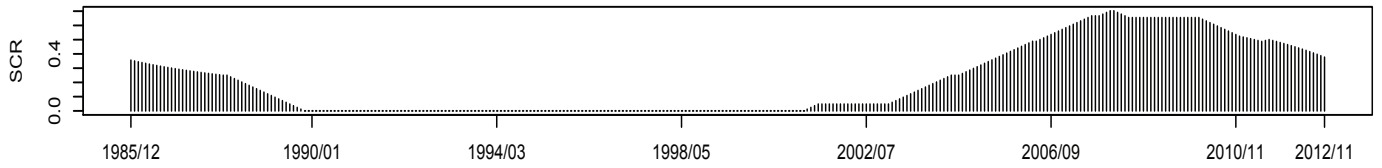

Causality running from KOSPI to HS

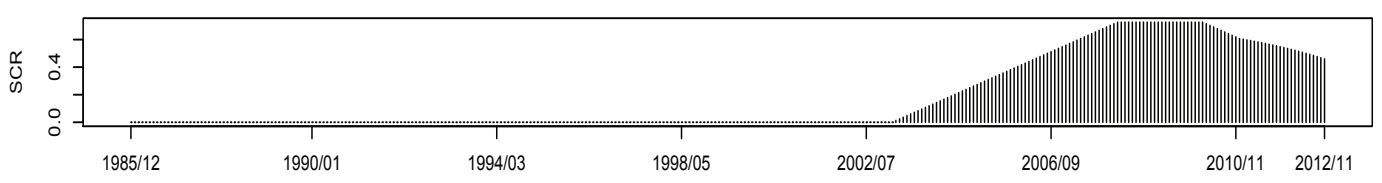

Figure 5: Causality running from KOSPI with a 7-year rolling window size. The graphs show the significant causality ratio that run from the KOSPI market to major stock markets in each month.

\section{Acknowledgments}

This work was supported by the National Research Foundation of Korea (NRF) grant funded by the Korea government (MSIT) (NRF-2018R1C1B5043739). This work was supported by Hankuk University of Foreign Studies Research Fund.

\section{References}

Ajmi AN, Hammoudeh S, Nguyen DK, and Sarafrazi S (2014). How strong are the causal relationships between Islamic stock markets and conventional financial systems? Evidence from linear and nonlinear tests, Journal of International Financial Markets, Institutions and Money, 28, 213-227.

Baek EA and Oh MS (2016). Volatility spillover between the Korean KOSPI and the Hong Kong HSI stock markets, Communications for Statistical Applications and Methods, 23, 203-213. 
Engle R (2002). Dynamic conditional correlation: a simple class of multivariate generalized autoregressive conditional heteroskedasticity models, Journal of Business \& Economic Statistics, 20, 339-350.

Eun CS and Shim S (1989). International transmission of stock market movements, Journal of Financial and Quantitative Analysis, 24, 241-256.

Granger CWJ (1969). Investigating causal relations by econometric models and cross-spectral methods, Econometrica: Journal of the Econometric Society, 37, 424-438.

Hafner CM and Herwartz H (2009). Testing for linear vector autoregressive dynamics under multivariate generalized autoregressive heteroskedasticity, Statistica Neerlandica, 63, 294-323.

Kim SJ (2005). Information leadership in the advanced Asia-Pacific stock markets: Return, volatility and volume information spillovers from the US and Japan, Journal of the Japanese and International Economies, 19, 338-365.

Kim SW and Rogers JH (1995). International stock price spillovers and market liberalization: Evidence from Korea, Japan, and the United States, Journal of Empirical Finance, 2, 117-133.

Kim WH (2014). Time-varying comovement of KOSPI 200 sector indices returns, Communications for Statistical Applications and Methods, 21, 335-347.

Kim WH and Bang SB (2014). Regime-dependent characteristics of KOSPI return, Communications for Statistical Applications and Methods, 21, 501-512.

Mantalos P (2000). A graphical investigation of the size and power of the Granger-causality tests in integrated-cointegrated VAR systems, Studies in Nonlinear Dynamics \& Econometrics, 4, 15583708.

Masih AMM and Masih R (1999). Are Asian stock market fluctuations due mainly to intra-regional contagion effects? Evidence based on Asian emerging stock markets, Pacific-Basin Finance Journal, 7, 251-282.

Masih R and Masih AMM (2001). Long and short term dynamic causal transmission amongst international stock markets, Journal of International Money and Finance, 20, 563-587.

McMillin WD (1988). Money growth volatility and the macroeconomy, Journal of Money, Credit and Banking, 20, 319-335.

Nyakabawo W, Miller SM, Balcilar M, Das S, and Gupta R (2015). Temporal causality between house prices and output in the US: A bootstrap rolling-window approach, The North American Journal of Economics and Finance, 33, 55-73.

Pesaran MH and Timmermann A (2005). Small sample properties of forecasts from autoregressive models under structural breaks, Journal of Econometrics, 129, 183-217.

Rapach DE, Strauss JK, and Zhou G (2013). International stock return predictability: what is the role of the United States?, The Journal of Finance, 68, 1633-1662.

Singh RP and Kishor N (2017). Short and long run inter linkages of market returns of Indian stock market with developed stock markets, International Journal of Technology Transfer and Commercialisation, 15, 203-223.

Smith KL, Brocato J, and Rogers JE (1993). Regularities in the data between major equity markets: evidence from Granger causality tests, Applied Financial Economics, 3, 55-60.

Stambaugh RF (1999). Predictive regressions, Journal of Financial Economics, 54, 375-421.

Yang T and Lim JJ (2004). Crisis, contagion, and East Asian stock markets, Review of Pacific Basin Financial Markets and Policies, 7, 119-151. 
